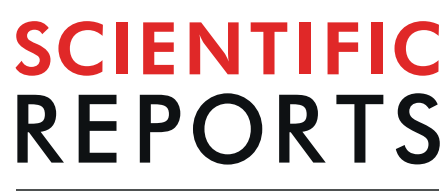

natureresearch

Check for updates

\title{
Clinically relevant mutations in mycobacterial LepA cause rifampicin-specific phenotypic resistance
}

\begin{abstract}
Bi-Wei Wang ${ }^{1,4}$, Jun-Hao Zhu ${ }^{1,2,4} \&$ Babak Javid ${ }^{1,3 凶}$
Although all wild-type bacterial populations exhibit antibiotic tolerance, bacterial mutants with higher or lower tolerant subpopulation sizes have been described. We recently showed that in mycobacteria, phenotypically-resistant subpopulations can grow in bulk-lethal concentrations of rifampicin, a first-line anti-tuberculous antibiotic targeting RNA polymerase. Phenotypic resistance was partly mediated by paradoxical upregulation of RNA polymerase in response to rifampicin. However, naturally occurring mutations that increase tolerance via this mechanism had not been previously described. Here, we used transposon insertional mutagenesis and deep sequencing (Tnseq) to investigate rifampicin-specific phenotypic resistance using two different in vitro models of rifampicin tolerance in Mycobacterium smegmatis. We identify multiple genetic factors that mediate susceptibility to rifampicin. Disruption of one gene, lepA, a translation-associated elongation factor, increased rifampicin tolerance in all experimental conditions. Deletion of lepA increased the subpopulation size that is able to grow in bulklethal rifampicin concentrations via upregulation of basal rpoB expression. Moreover, homologous mutations in lepA that are found in clinical Mycobacterium tuberculosis (Mtb) isolates phenocopy lepA deletion to varying degrees. Our study identifies multiple genetic factors associated with rifampicin tolerance in mycobacteria, and may allow correlation of genetic diversity of clinical Mtb isolates with clinically important phenotypes such as treatment regimen duration.
\end{abstract}

Antibiotic tolerance describes genetically susceptible bacterial subpopulations that are killed more slowly than the bulk population ${ }^{1,2}$. There are a spectrum of phenotypes associated with antibiotic tolerance ${ }^{3}$. The best studied is non-replicating persistence - in which non- or slowly-replicating bacteria are typically multi-drug tolerant ${ }^{1}$. However, increasing evidence, particularly in mycobacteria, suggests that actively replicating bacteria can also be highly drug tolerant ${ }^{4-9}$. We have previously focused on tolerance in actively growing cells to the first-line anti-tuberculous antibiotic rifampicin, which inhibits RNA polymerase (RNAP), and which we termed rifampicin-specific phenotypic resistance (RSPR). We observed that mycobacteria can not only survive, but actively grow in bulk-lethal concentrations of rifampicin. Both specific translational errors involving the indirect tRNA aminoacylation pathway, as well as a paradoxical upregulation of $r p o B$ in response to rifampicin mediated $\mathrm{RSPR}^{7,9,10}$. Importantly, both mechanisms of RSPR were confirmed in clinical isolates of Mycobacterium tuberculosis (Mtb), corroborating the potential clinical relevance of this form of antibiotic tolerance ${ }^{7,9}$. Transposon insertion mutagenesis and deep sequencing (Tnseq) has proven a valuable tool for forward genetics in bacteria. Although it has been used extensively for identification of genetic factors involved in bacterial physiology, host-pathogen interactions, as well as antibiotic resistance ${ }^{11-15}$, investigation of antibiotic tolerance in mycobacteria using Tnseq has been limited ${ }^{16-18}$. Here, we use Tnseq in two models of rifampicin tolerance in Mycobacterium smegmatis (Msm) and identify genetic factors implicated in both hypertolerance and hypersusceptibility to rifampicin. In particular, we identify that deletion of the putative translation elongation factor LepA mediates RSPR via perturbation of the physiological transcriptional response of $r p o B$, and show that mutations in lepA identified in clinical Mtb isolates phenocopy lepA deletion in mediating rifampicin tolerance. Given these

${ }^{1}$ Centre for Global Health and Infectious Diseases, Collaborative Innovation Centre for the Diagnosis and Treatment of Infectious Diseases, Tsinghua University School of Medicine, Beijing, China. ${ }^{2}$ Immunology and Infectious Diseases, Harvard TH Chan School of Public Health, Boston, USA. ${ }^{3}$ Beijing Advanced Innovation Center in Structural Biology, Beijing, China. ${ }^{4}$ These authors contributed equally: Bi-Wei Wang and Jun-Hao Zhu. ${ }^{\bowtie e-m a i l: ~ b j a v i d @ g m a i l . c o m ~}$ 
mutations are in conserved sites between M. smegmatis and M. tuberculosis, it is likely their phenotypes will be conserved.

\section{Results}

Tnseq identifies inactivation of lepA as a mediator of rifampicin phenotypic resistance. To investigate non-essential genes contributing to rifampicin-specific phenotypic resistance in Mycobacterium smegmatis, we first constructed a high density $\left(1.5 \times 10^{5}\right.$ unique clones $)$ transposon-mutagenized library via phage transduction of a Himar1 transposon that inserts at TA dinucleotides within the genome ${ }^{12,19}$. The library was subjected to 4 different selection conditions: plating on rifampicin-agar at either $10 \mu \mathrm{g} / \mathrm{mL}$ or $20 \mu \mathrm{g} / \mathrm{mL}$ (representing $1 \mathrm{x}$ and $2 \mathrm{x}$ the plating $\mathrm{MIC}_{90}$ ), or inoculation into $7 \mathrm{H} 9$ liquid medium containing $10 \mu \mathrm{g} / \mathrm{mL}$ or $20 \mu \mathrm{g} / \mathrm{mL}$ rifampicin (representing $4 \mathrm{x}$ and $8 \mathrm{x}$ the liquid culture $\mathrm{MIC}_{90}$ ). We had previously shown that under these conditions, selection resulted in survival of between $3-10 \%$ of inoculated bacteria ${ }^{9}$, thus allowing for analysis of survivors by Tnseq. Importantly, survival and growth-mediated tolerance on rifampicin-agar would exclude genetic mutants that induced a non-replicating persister state, allowing us to interrogate alternative mechanisms of tolerance involving actively growing bacteria. Following selection, bacteria were pooled, lysed and genomic DNA extracted, and transposon insertion site frequencies mapped by deep sequencing and compared with the input library prior to selection (Fig. 1a and ${ }^{12,19}$ ).

Rifampicin resistance is mediated solely by mutations in the rifampicin-resistance determining region of the $r p o B$ gene, coding for the essential $\beta$ subunit of RNAP. Thus, transposon mutagenesis, which targets non-essential genes only, would not select for bona fide rifampicin resistant mutants. Therefore our selection strategy would enrich for mutants that were either hyper-susceptible or hyper-tolerant (but not resistant) to rifampicin.

We compared transposon insertion sites that were both under- and over-represented under rifampicin selection by TRANSIT tool ${ }^{19}$ resampling with correction for multiple comparisons (Fig. 1b-e, Fig. S1 and Datasets S1 and S2). In total, transposon insertions in 34 genes were significantly under-represented under all four conditions of rifampicin selection, suggesting that they were conditionally essential for rifampicin tolerance (Fig. 1f and Table S1). By contrast, transposon insertions were over-represented in a single gene, lepA (Msmeg_4556) under all four conditions (Figs. $1 \mathrm{~g}$ and S2).

Mutations in lepA identified from clinical isolates confer rifampicin phenotypic resistance. Since transposon insertion in lepA was identified as a cause for increased rifampicin tolerance in all four experimental conditions, we decided to focus on deletion of lepA for further characterization. We constructed a strain of $M$. smegmatis in which the gene coding for lepA was deleted by recombineering ${ }^{7}, \Delta l e p A$. The minimum inhibitory concentration (MIC) to several anti-mycobacterial antibiotics was similar between wild-type M. smegmatis and $\triangle l e p A$, confirming that deletion of lepA did not confer altered resistance, including to rifampicin (Table S2). However, the strain lacking lepA had significantly greater survival to rifampicin compared with the wild-type parent strain and this phenotype was complemented with wild-type lepA (Fig. 2a). There was no increased tolerance to the antibiotics isoniazid or streptomycin (Fig. S3), suggesting that lepA deletion was not a cause of non-specific antibiotic tolerance. Of note, a substantial number of clinical isolates of $M$. tuberculosis harbor mutations in the lepA gene. We selected a number of mutations identified from clinical isolates (https://platform.reseqtb.org), mapping to conserved residues of the annotated GTP-binding domain of the protein (Dataset S1 and Fig. S4). We then complemented the $\Delta l e p A$ strain with either the wild-type or mutated lepA genes. Intriguingly, all of the conserved mutations failed to fully complement $\Delta l e p A$, but to variable extents (Fig. $2 \mathrm{~b}$ ), suggesting a degree of loss of function in LepA in a proportion of circulating clinical M. tuberculosis isolates.

LepA deletion increases basal expression of RpoB and blunts rifampicin-induced RSPR. We had previously shown that rifampicin and other inhibitors of RNAP triggered upregulation of $r p o B-r p o C$ expression due to the operon-specific promoter architecture. This paradoxical upregulation in $r p o B$ - $r p o C$ expression was stringently associated with growing tolerant mycobacteria ${ }^{9}$. We wondered whether deletion of lepA was associated with an aberrant response to this tolerance-associated transcriptional response. Using a previously characterized fluorescent reporter, $\mathrm{P}_{(\mathrm{rpoB}-\mathrm{rpoC})}-\mathrm{mEmerald}$, which measures the transcriptional response from the $r p o B-r p o C$ promoter, we observed that deletion of lepA resulted in a higher 'basal' degree of expression from the promoter, but addition of subinhibitory concentrations of rifampicin failed to further upregulate expression (Fig. 2c). We had previously demonstrated that the mycobacterial $r p o B$ - $r p o C$ promoter contains two conserved promoters. Expression from the $5^{\prime}$ promoter I dampened the maximal response from the stronger promoter II $^{9}$. Deletion of promoter I or its inhibition by subinhibitory concentrations of rifampicin relieved inhibition of promoter II and caused upregulation of $r p o B$-rpoC expression and hence increased rifampicin tolerance ${ }^{9}$. The lepA deletion strain behaved similarly to a strain in which promoter I had been deleted. We therefore hypothesized that deletion of lepA would dampen rifampicin-induced rifampicin-specific phenotypic resistance. We used two complementary assays for grower-mediated rifampicin tolerance ${ }^{9}$ to test this hypothesis. In the first assay, the mycobacterial cell wall is fluorescently labelled by a fluorophore (Alexafluor-488), and cells are grown in the presence of bactericidal concentrations of drug. Cells that are nonetheless able to survive and grow in bulk-lethal concentrations of drug will lose fluorescence due to fluorescence-dilution, allowing measurement of the 'grower' population ${ }^{9}$. As previously demonstrated ${ }^{9}$, in wild-type M. smegmatis, exposure to sublethal concentrations of rifampicin prior to exposure to bulk-lethal concentrations of rifampicin led to significantly increased RSPR. However, although the lepA-deletion strain had higher basal rifampicin tolerance, this did not increase upon pre-exposure to sublethal rifampicin (Fig. 2 d), and this phenotype was complementable. We also verified the phenotype in the 'plate-growth' assay used in the initial screening conditions. Exposure of wild-type M. smegmatis to sublethal rifampicin prior to plating on rifampicin-agar $(10 \mu \mathrm{g} / \mathrm{ml})$ led to 10 -fold increase in RSPR, with a much more blunted response in a strain deleted for lepA (Fig. 2e). Together, these assays demonstrated that lepA 
a.
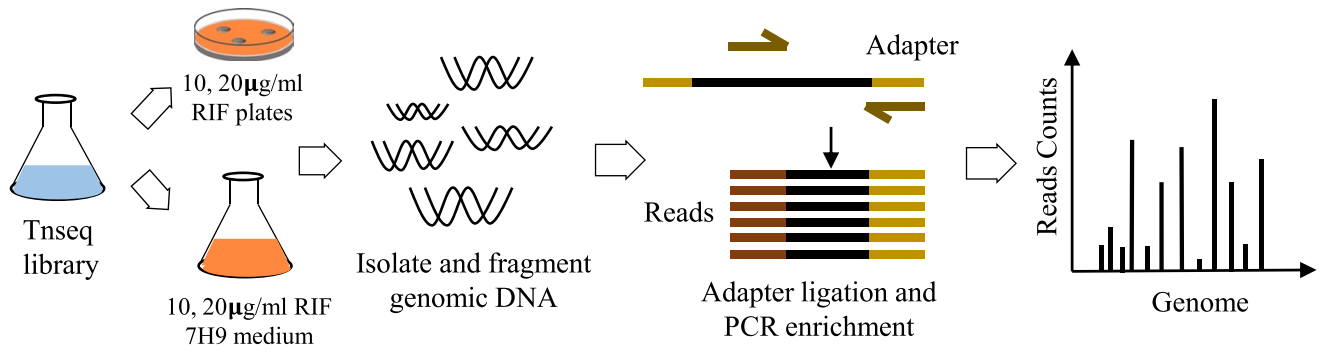

b.

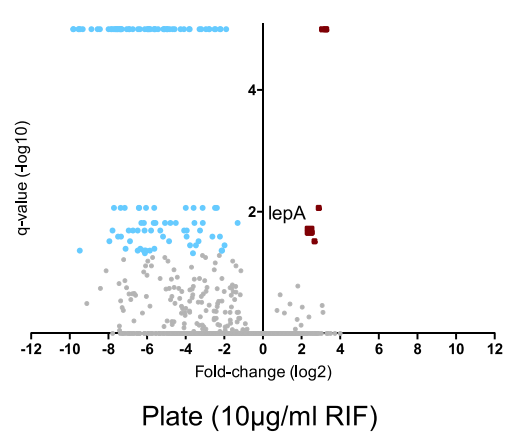

c.

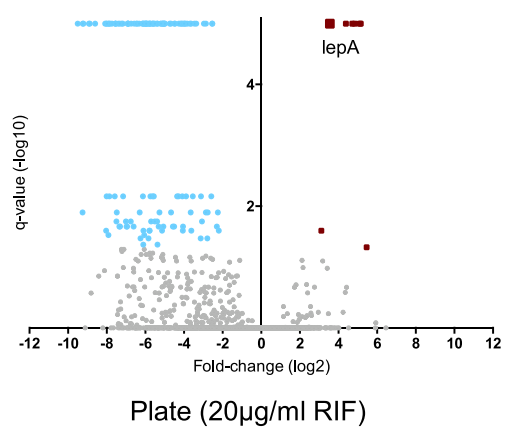

d.

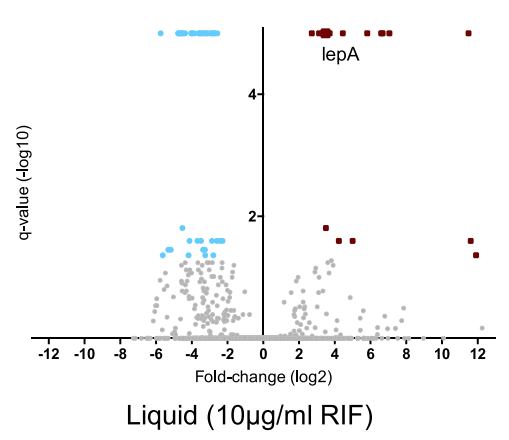

e.

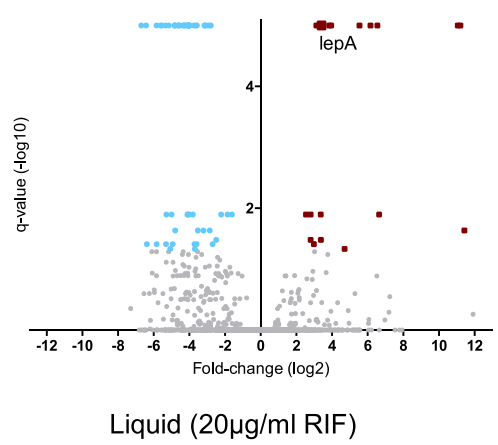

f.

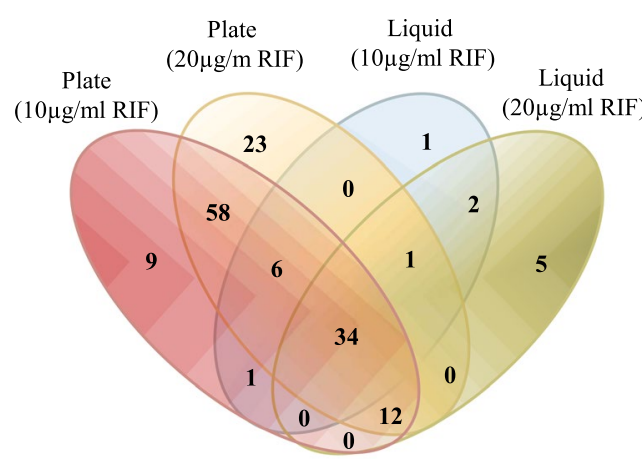

Fold-change $<0.5$

g.
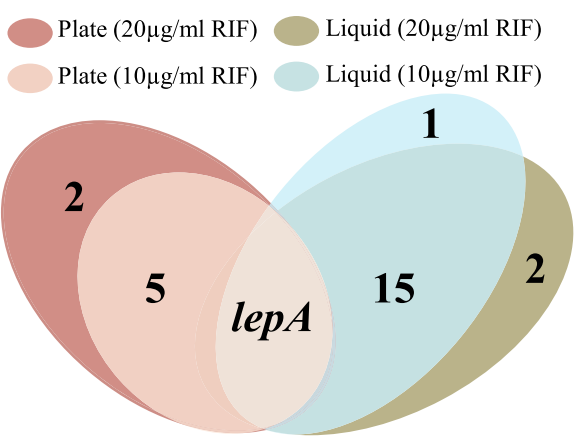

Fold-change $>2$

Figure 1. Tnseq identifies genes contributing to rifampicin-specific phenotypic resistance. (a) Cartoon illustrating selection strategy. A transposon-insertion mutagenized library of Mycobacterium smegmatis (Msm Tn library) was subjected to four different selection conditions as illustrated, following which genomic DNA of survivors was extracted and prepared for transposon insertion site mapping by deep sequencing (see Methods). Volcano plots illustrating significantly enriched Tn reads under selection by rifampicin-agar $10 \mu \mathrm{g} / \mathrm{mL}(\mathbf{b})$, or $20 \mu \mathrm{g} / \mathrm{mL}$ (c), or 7H9-rifampicin $10 \mu \mathrm{g} / \mathrm{mL}$ (d) or $20 \mu \mathrm{g} / \mathrm{mL}$ (e) after resampling by TRANSIT tool. Genes with insertions enriched $\log _{2}(<0.5)$ compared with the input library and q-value $<0.05$ are shown with blue dots and those enriched $\log _{2}(>2)$ and q-value $<0.05$ are shown as red dots. lepA(Msmeg_4556) is highlighted. See also Datasets 1 and 2. Venn diagrams illustrating overlap in enriched for under-represented (f) and overrepresented $(\mathbf{g}) \mathrm{Tn}$ insertion reads in the four conditions. 
a.

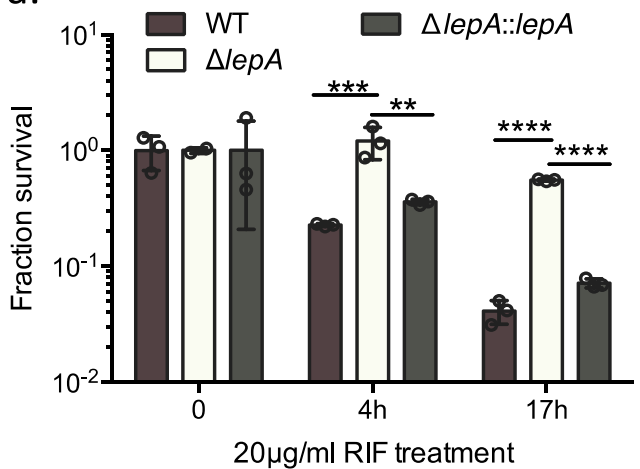

C.

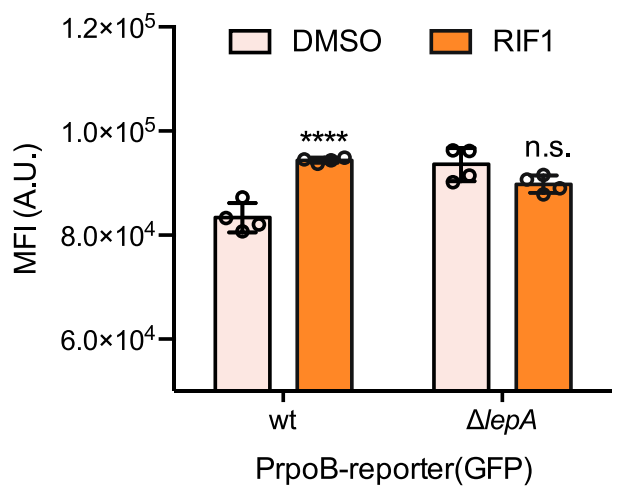

e.

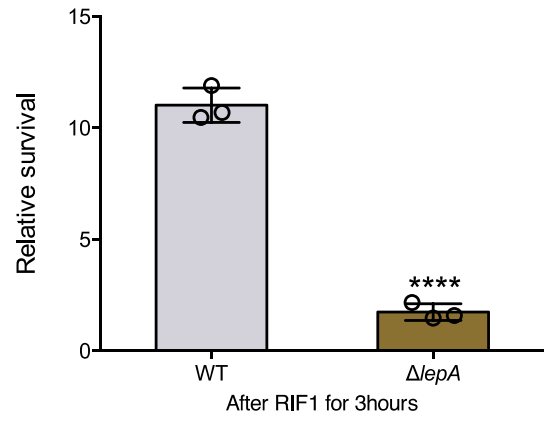

b.

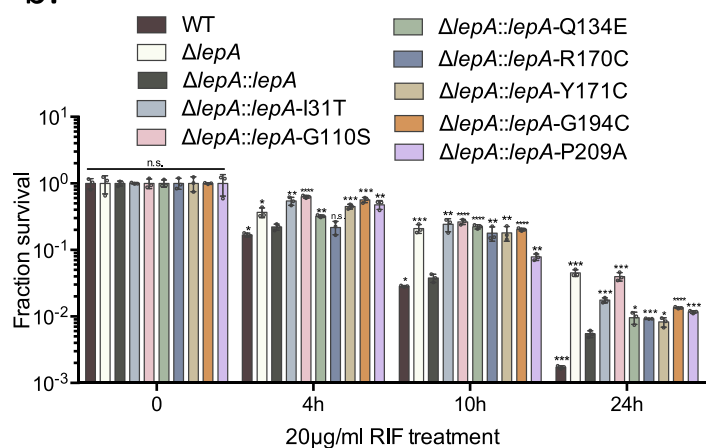

d.

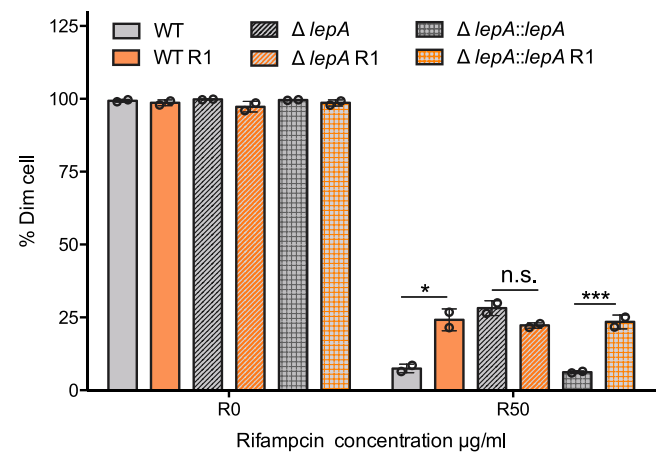

Figure 2. Loss of function in LepA causes rifampicin-specific phenotypic resistance. (a) Relative survival of wild-type (WT) M.smegmatis (Msm) following treatment with $20 \mu \mathrm{g} / \mathrm{mL}$ rifampicin in axenic culture compared with the lepA deletion strain $(\triangle l e p A)$ and the deletion strain complemented with wild-type lepA $(\Delta l e p A:: l e p A)$. (b) Relative survival in rifampicin of WTM. smegmatis compared with the lepA deletion strain $(\triangle l e p A)$, or the deletion strain complemented with wild-type lepA $(\Delta l e p A:: l e p A)$ or specified mutations in lepA. Statistical comparison of means with $\triangle l e p A:: l e p A$ by Student's t-test is shown. (c) Relative green fluorescence, representing expression from the $r p o B$-rpoC-promoter driven GFP of WT Msm and $\Delta l e p$ Astrains. (d) WT or $\Delta l e p A$ strains were treated with $1 \mu \mathrm{g} / \mathrm{mL}$ rifampicin or carrier for 3hours, following which the bacteria were stained with AF488 and then treated with rifampicin at indicated concentrations or carrier (DMSO) overnight, and proportion of dim cells, representing growing bacteria plotted. (e) WT or $\Delta l e p A$ strains were treated with $1 \mu \mathrm{g} / \mathrm{mL}$ rifampicin or carrier for 3 hours, following which bacteria were pelleted, washed and replated on rifampicin-agar $10 \mu \mathrm{g} / \mathrm{mL}$ or non-selective medium. Relative survival of RIF 1-treated compared with untreated bacteria are shown. ${ }^{*} \mathrm{p}<0.05, * * \mathrm{p}<0.01, * * * \mathrm{p}<0.001$ and ${ }^{* * * *} \mathrm{p}<0.0001$ by Student's t-test. n.s. no significant difference.

deletion phenocopied $r p o B-C$ promoter I deletion, resulting in increased basal rifampicin tolerance, but without a subsequent increase due to rifampicin exposure.

\section{Discussion}

Our study leverages the power of forward genetics using transposon site insertion and deep sequencing (Tnseq) to identify non-essential mycobacterial genes that cause rifampicin phenotypic resistance. Our screen identified a number of genes previously associated with antibiotic tolerance in mycobacteria, as well as new hits. A number of our hits are in proteins embedded in the cell wall/outer mycobacterial layer and/or implicated in cell-wall 
integrity, such as MmpL11, Antigen85A, PstS, M_SMEG5782c and LytR. Of these, several have been previously identified as associated with either non-replicating persistence and/or biofilm formation ${ }^{20-23}$. Cellular processes that disrupt cell wall integrity and therefore increase intra-cellular antibiotic concentrations would be expected to increase antibiotic susceptibility across many different antibiotics, and this has been verified for the phosphate transporter $\mathrm{Pst}^{24}$. The serine/threonine protein kinase, PknG had also been previously identified as important for mycobacterial adaptation to acid stress and persistence ${ }^{25,26}$. In addition to hits implicated in cell wall integrity and/or environmental sensing, our screen identified a number of genes involved in carbon metabolism, for example ArgG, but in particular for branched-chain amino acid synthesis such as IlvB, IlvE and MetH (Table S1). Although it is conceivable that inhibition of central carbon metabolism and/or branched chain amino acid synthesis might result in a state of non-replicating persistence and multi-drug tolerance, these hits were also identified under the rifampicin-agar plate growth conditions that would have specifically excluded persisters. Therefore the exact mechanism by which disruption of these genes increases rifampicin tolerance without necessarily compromising growth in antibiotics is not known.

We chose to focus on LepA in our study, since that was the only gene in which transposon disruption increased rifampicin tolerance instead of increasing susceptibility under all four experimental conditions. LepA is a highly conserved GTPase, with sequence similarity to EF-G, a translation-associated elongation factor, but the precise cellular function of LepA remains controversial ${ }^{27-29}$. LepA was also identified in another forward genetic screen that specifically investigated intra-cellular concentrations of the fluorescent dye calcein, which was in turn associated with rifampicin tolerance ${ }^{14}$. A recent preprint implicates LepA in the synthesis of a mycobacterial porin, and in turn rifampicin and vancomycin tolerance ${ }^{30}$. However, both our data and that of Rubin et al. suggests that lepA deletion has potentially wider-ranging perturbations of transcriptional and translational responses. Specifically, our data identifying that the lepA deletion mutant has a blunted response to sublethal rifampicin exposure, which phenocopies rpoB-rpoC promoter I deletion ${ }^{9}$ suggests that the rifampicin tolerance phenotype may involve multiple, non-mutually exclusive mechanisms.

A potential drawback of transposon mutagenesis screens is that hits only in non-essential genes can be identified. However, loss of function mutations in clinical strains are more likely to occur in these non-essential genes, and therefore identification of clinically-relevant phenotypes associated with such loss of function mutations ${ }^{31,32}$ may allow for patient-specific therapeutic regimens.

\section{Materials and Methods}

Bacterial culture. Mycobacterium smegmatis $\mathrm{mc}^{2}-155$ (ATCC) was grown in liquid Middlebrook $7 \mathrm{H} 9$ broth (BD) containing $0.5 \%$ glycerol, $0.05 \%$ Tween $80,10 \%$ (ADS, albumin-dextrose-salt) or on LB (BD) agar at $37^{\circ} \mathrm{C}$. Escherichia coli was cultured in LB broth Miller (BD) or on LB agar plates at $37^{\circ} \mathrm{C}$. Where needed, antibiotics were used at the following concentrations: kanamycin $(25 \mu \mathrm{g} / \mathrm{ml}$ for $M$. smegmatis and $50 \mu \mathrm{g} / \mathrm{ml}$ for E. coli), zeocin $(25 \mu \mathrm{g} / \mathrm{ml}$ for M. smegmatis and $50 \mu \mathrm{g} / \mathrm{ml}$ for E. coli), hygromycin $(75 \mu \mathrm{g} / \mathrm{ml}$ for M. smegmatis and $150 \mu \mathrm{g} / \mathrm{ml}$ for $E$. coli). Rifampicin (TCI) was dissolved in DMSO (stock concentration as $30 \mathrm{mg} / \mathrm{ml}$ ) and added to culture medium at indicated concentrations.

Strain construction. All primers used in this study are listed in Table S3. The $\Delta l e p A$ strain was constructed using Rec-ET homologous recombination system as previously reported ${ }^{7}$. Briefly, a 515 bp DNA fragment upstream of lepA (MSMEG_4556) was PCR amplified using primers MSMEG_4556_KO_1 and MSMEG_4556_ KO_2. Similarly, a downstream 507 bp fragment was amplified using MSMEG_4556_KO_3 and MSMEG_4556_ KO_4. A Zeocin resistant marker flanked by two LoxP sites was amplified from a template plasmid pKM_Zeo_lox (a kind gift from Dr. Eric Rubin) using Zeo_F and Zeo_R. The three PCR products were stitched together through PCR with primers MSMEG_4556_KO_1 and MSMEG_4556_KO_4. Fresh Rec-ET expressing competent cells were prepared as previously described, transformed with 2 micrograms of purified stitch-PCR product, and selected on LB agar with $25 \mu \mathrm{g} / \mathrm{ml}$ Zeocin. The L5 site integrating plasmid pML1342 system ${ }^{33}$ was employed to construct complementation strains. Wild-type lepA was amplified with primers HindIII_lepA_F and XbaI_lepA_R from M. smegmatis genomic DNA by KOD DNA Polymerase (NEB). The lepA fragment and pML1342 were digested with restriction endonucleases XbaI and HindIII (NEB). After gel purification using V-RLUTE Gel Mini Purification Kit (ZOMANBIO) fragments were ligated with T4 DNA Ligase (NEB) to obtain plasmid pML_1342_ lepA. Plasmids pML_1342_SNPlepA1-7 were constructed with forward primers SNP170_F - SNP682_F and universal reverse primer SNP170_R - SNP682_R using pML_1342_lepA as template. Plasmids pML_1342_ SNPlepA1-7 were transformed into E.coli DH5 $\alpha$ competent cells (CW Biotech) using $150 \mu \mathrm{g} / \mathrm{ml}$ hygromycin for selection. Recombinant plasmids pML1342 containing wildtype lepA and mutated lepA were transformed into fresh $\Delta l e p A$ competent cells by electroporation equipment (Bio-Rad) with voltage $2500 \mathrm{~V}$, capacitance $25 \mu \mathrm{F}$, resistance $400 \Omega$. M. smegmatis was selected on LB agar plates containing $40 \mu \mathrm{g} / \mathrm{ml}$ hygromycin - see Fig. S5 for PCR validation. The wild-type lepA or individual mutated lepA genes were integrated into the M. smegmatis genome at mycobateriophage L5 attachment site attB. The success of strain construction was confirmed by sequenced results of lepA PCR products using mutant strains as templates.

Transposon mutagenesis library construction. The M. smegmatis transposon mutant library was constructed according to reference ${ }^{12}$ with minor changes. Briefly, $100 \mathrm{ml}$ exponentially growing $\left(\mathrm{OD}_{600 \mathrm{~nm}}=0.6-0.8\right)$ M. smegmatis culture was washed three times with MP buffer $(50 \mathrm{mM}$ Tris $\mathrm{HCl}, \mathrm{pH} 7.5,150 \mathrm{mM} \mathrm{NaCl}, 10 \mathrm{mM}$ $\mathrm{Mg}_{2} \mathrm{SO}_{4}, 2 \mathrm{mM} \mathrm{CaCl}_{2}$ ) to remove excess culture medium, and re-suspended in $15 \mathrm{ml} \mathrm{MP}$ buffer mixed with freshly prepared phage $\left(2 \times 10^{11}\right.$ plaque forming units). The transduction mixture was incubated at $37^{\circ} \mathrm{C}$ for three hours with gentle agitation. $3 \mathrm{ml}$ aliquots were then plated onto each of the $5 \mathrm{LB}$ agar plates (prepared in $25 \times 25 \mathrm{~cm}$ square petri dishes) containing $20 \mu \mathrm{g} / \mathrm{ml} \mathrm{Kanamycin} \mathrm{and} 0.05 \%$ Tween 80 . After 3 days of incubation at $37^{\circ} \mathrm{C}$, 
colonies were scraped off the plates and mixed in $20 \mathrm{~mL} 7 \mathrm{H} 9$ broth with $15 \%$ glycerol. $2 \mathrm{ml}$ of the mixture (input library) were immediately subjected to gDNA extraction, while the remaining was kept at $-80^{\circ} \mathrm{C}$ as $1 \mathrm{ml}$ aliquots.

Rifampicin selection. Two of the $1 \mathrm{ml}$ frozen transposon library stocks were thawed on ice, and recovered in $50 \mathrm{ml}$ fresh $7 \mathrm{H} 9$ medium for 3 hours at $37^{\circ} \mathrm{C}$. For agar-plate based selection, aliquots of the recovered library containing $5 \times 10^{4}$ or $2 \times 10^{5}$ bacteria were plated on each of 20 agar plates (prepared in $15 \mathrm{~cm} \times 15 \mathrm{~cm}$ round petri dishes) supplemented with $10 \mu \mathrm{g} / \mathrm{ml}$ or $20 \mu \mathrm{g} / \mathrm{ml}$ rifampicin, respectively. For selection in liquid culture, $10^{9}$ bacteria from the recovered library were pelleted and inoculated into flasks containing $100 \mathrm{ml}$ fresh $7 \mathrm{H} 9 \mathrm{medium}$ and $10 \mu \mathrm{g} /$ $\mathrm{ml}$ or $20 \mu \mathrm{g} / \mathrm{ml} \mathrm{rifampicin.} \mathrm{All} \mathrm{agar} \mathrm{plates} \mathrm{were} \mathrm{covered} \mathrm{in} \mathrm{foil} \mathrm{and} \mathrm{kept} \mathrm{at} 37^{\circ} \mathrm{C}$. After 5 days $(1$ more day after colonies became visible), colonies from each selection condition were scraped off the plates, washed once with fresh 7H9 medium and once with TE buffer ( $10 \mathrm{mM}$ Tris- $\mathrm{HCl}, 1 \mathrm{mM}$ EDTA at $\mathrm{pH}$ 9), then re-suspended in $2 \mathrm{ml} \mathrm{TE} \mathrm{buffer.} \mathrm{The}$ two liquid cultures were kept at $37^{\circ} \mathrm{C}$ with constant shaking (200 rpm) for 36 hours, washed twice with TE buffer, then re-suspended in $2 \mathrm{ml} \mathrm{TE}$ buffer. The four samples were stored at $-80^{\circ} \mathrm{C}$ for further genomic DNA extraction.

Transposon sequencing library preparation. Genomic DNA extraction and sequencing library construction were conducted as described in reference ${ }^{34}$. DNA concentrations of the PCR-amplified pre-sequencing samples were quantified using Qubit 2.0 (dsDNA, High-sensitivity kit, Invitrogen) and adjusted to the same concentration using nuclease-free water. The libraries were then pooled and subjected to high-throughput sequencing with the Miseq platform according to manufacturer's instructions.

Sequencing data analysis. Raw data was downloaded from MiSeq local server and decompressed before further analysis. TPP pipeline from TRANSIT ${ }^{19}$ was used for pre-processing of raw data, and processed reads were mapped to the M. smegmatis mc $^{2}-155$ genome (GenBank accession number GCA_000015005.1) using BWA (Burroughs-Wheeler Aligner). Read1 and Read2 were used to match the terminus of the Himar 1 transposon and to extract random barcodes respectively. Unique "template" counts at each TA sites were generated as wig formula. Resampling test module from TRANSIT was used in testing gene conditional fitness cost under each selection condition, which is a classical permutation test in statistics. TTR (trimmed total reads) was used as the normalization method in resampling. Significance of differences between each conditional selection and non-selection was represented by log2-fold change through comparison with a resampling distribution that is generated from randomly reshuffling of the observed counts at TA sites in the region among all datasets. Relative $\mathrm{P}$-value were generated within $10^{4}$ permutations according to the proportion of reshuffling samples that have more significant difference than in the actual experimental data.

RSPR killing curve assay. Wild type M. smegmatis or its derivatives were cultured in 7H9 liquid medium until exponential growth phase i.e. optical density $\left(\mathrm{OD}_{600 \mathrm{~nm}}\right)$ reached $0.6-0.8$. The cultures were then diluted in $7 \mathrm{H} 9$ medium to final $\mathrm{OD}_{600 \mathrm{~nm}}=0.05$, and supplemented with rifampicin at indicated concentrations. Samples from each treatment were taken at 0 hour, 4 hours and 17 hours after inoculation, washed and subjected to several $10 \times$ dilutions, then plated on antibiotic-free LB plates. Fractional survival was estimated as previously described ${ }^{9}$.

Rif-induced RSPR plating assay. Wild-type M. smegmatis, or its derivatives were cultured in 7H9 liquid medium until optical density $\left(\mathrm{OD}_{600 \mathrm{~nm}}\right)$ reached $0.6-0.8 .1 \mathrm{ml}$ bacteria was inoculated in $7 \mathrm{H} 9$ medium with $1 \mu \mathrm{g} /$ $\mathrm{ml}$ rifampicin or carrier (DMSO). After 3 hours treatment, bacteria were collected and washed with PBST (PBS containing $0.05 \%$ tween 80 ) and several $10 \times$ dilutions were plated on antibiotic-free or rifampicin agar plates as indicated. RSPR was calculated by the ratio of CFU between plates with and without rifampicin. Relative survival represents the fold-change of rifampicin tolerance with $1 \mu \mathrm{g} / \mathrm{ml}$ rifampicin pre-treatment.

Alexa-fluor-488 (AF488) assay. The AF488 assay was adapted from reference ${ }^{9}$ with some modifications. SNAP-Surface Alexa Fluor 488 (NEB) was dissolved in DMSO for $5 \mathrm{mg} / \mathrm{ml}$ stock solution and stored at $-80^{\circ} \mathrm{C}$ for up to several weeks. $2 \mathrm{ml}$ of the strain to be tested was cultured in $7 \mathrm{H} 9$ medium until exponential growth phase with optical density $\left(\mathrm{OD}_{600 \mathrm{~nm}}=0.6-0.8\right) .1 \mathrm{ml}$ bacteria were inoculated in $7 \mathrm{H} 9$ medium with and without $1 \mu \mathrm{g} / \mathrm{ml}$ rifampicin respectively for 3 hours. After two washes with $1 \mathrm{ml}$ PBST, bacteria was re-suspended in $50 \mu \mathrm{l}$ diluted AF488 (working concentration $200 \mu \mathrm{g} / \mathrm{ml}$ ). After incubating in the dark at room temperature for $5 \mathrm{~min}$, bacteria were transferred into a fresh tube and then washed twice more with $1 \mathrm{ml}$ PBST. Bacterial pellets were re-suspended in $500 \mu \mathrm{l} 7 \mathrm{H} 9$ fresh medium. $100 \mu \mathrm{l}$ of the suspension were inoculated into $7 \mathrm{H} 9$ medium containing $0 \mu \mathrm{g} / \mathrm{ml}, 10 \mu \mathrm{g} / \mathrm{ml}, 50 \mu \mathrm{g} / \mathrm{ml}$ rifampicin. After 16 hours culture in $37^{\circ} \mathrm{C}$ shaking incubator in the dark, $100 \mathrm{ul}$ samples were fixed by $100 \mathrm{ul} 4 \%$ Paraformaldehyde (PFA) at room temperature for $20 \mathrm{~min}$. BD Accuri C6 desktop flow cytometry was used to collect fluorescence intensity with $488 \mathrm{~nm}$ excitation laser and $533 \mathrm{~nm}$ emission filter. $\%$ Dim cells representing the relative percentage of growing cells were analyzed by Flow-Jo software.

Statistical tests. All experiments were repeated in at least 3 times independently. Data are shown as means \pm SD. Differences in means were calculated using Student's t-test.

Received: 7 January 2020; Accepted: 28 April 2020;

Published online: 21 May 2020

\section{References}

1. Brauner, A., Fridman, O., Gefen, O. \& Balaban, N. Q. Distinguishing between resistance, tolerance and persistence to antibiotic treatment. Nat. Rev. Microbiol. 14, 320-330, https://doi.org/10.1038/nrmicro.2016.34 (2016)

2. Maisonneuve, E. \& Gerdes, K. Molecular mechanisms underlying bacterial persisters. Cell 157, 539-548, https://doi.org/10.1016/j. cell.2014.02.050 (2014) 
3. Aldridge, B. B., Keren, I. \& Fortune, S. M. The Spectrum of Drug Susceptibility in Mycobacteria. Microbiology spectrum 2, https:// doi.org/10.1128/microbiolspec.MGM2-0031-2013 (2014).

4. Aldridge, B. B. et al. Asymmetry and aging of mycobacterial cells lead to variable growth and antibiotic susceptibility. Science 335, 100-104, doi:10.1126/science.1216166science.1216166 [pii] (2012).

5. Javid, B. et al. Mycobacterial mistranslation is necessary and sufficient for rifampicin phenotypic resistance. Proc. Natl Acad. Sci. USA 111, 1132-1137, https://doi.org/10.1073/pnas.1317580111 (2014).

6. Richardson, K. et al. Temporal and intrinsic factors of rifampicin tolerance in mycobacteria. Proc. Natl Acad. Sci. USA 113, 8302-8307, https://doi.org/10.1073/pnas.1600372113 (2016).

7. Su, H. W. et al. The essential mycobacterial amidotransferase GatCAB is a modulator of specific translational fidelity. Nat. Microbiol. 1, 16147, https://doi.org/10.1038/nmicrobiol.2016.147 (2016).

8. Wakamoto, Y. et al. Dynamic persistence of antibiotic-stressed mycobacteria. Science 339, 91-95, doi:10.1126/science.1229858339/6115/91 [pii] (2013).

9. Zhu, J. H. et al. Rifampicin can induce antibiotic tolerance in mycobacteria via paradoxical changes in rpoB transcription. Nat. Commun. 9, 4218, https://doi.org/10.1038/s41467-018-06667-3 (2018).

10. Chaudhuri, S. et al. Kasugamycin potentiates rifampicin and limits emergence of resistance in Mycobacterium tuberculosis by specifically decreasing mycobacterial mistranslation. Elife 7, https://doi.org/10.7554/eLife.36782 (2018).

11. Carey, A. F. et al. TnSeq of Mycobacterium tuberculosis clinical isolates reveals strain-specific antibiotic liabilities. PLoS Pathog. 14, e1006939, https://doi.org/10.1371/journal.ppat.1006939 (2018).

12. DeJesus, M. A. et al. Comprehensive Essentiality Analysis of the Mycobacterium tuberculosis Genome via Saturating Transposon Mutagenesis. MBio 8, https://doi.org/10.1128/mBio.02133-16 (2017).

13. Griffin, J. E. et al. High-resolution phenotypic profiling defines genes essential for mycobacterial growth and cholesterol catabolism. PLoS Pathog. 7, e1002251, https://doi.org/10.1371/journal.ppat.1002251 (2011).

14. Rego, E. H., Audette, R. E. \& Rubin, E. J. Deletion of a mycobacterial divisome factor collapses single-cell phenotypic heterogeneity. Nature 546, 153-157, https://doi.org/10.1038/nature22361 (2017).

15. Zhang, Y. J. et al. Tryptophan biosynthesis protects mycobacteria from CD4 T-cell-mediated killing. Cell 155, 1296-1308, https:// doi.org/10.1016/j.cell.2013.10.045 (2013).

16. Bellerose, M. M. et al. Common Variants in the Glycerol Kinase Gene Reduce Tuberculosis Drug Efficacy. mBio 10, https://doi. org/10.1128/mBio.00663-19 (2019).

17. Rosen, B. C., Dillon, N. A., Peterson, N. D., Minato, Y. \& Baughn, A. D. Long-Chain Fatty Acyl Coenzyme A Ligase FadD2 Mediates Intrinsic Pyrazinamide Resistance in Mycobacterium tuberculosis. Antimicrob Agents Chemother 61, https://doi.org/10.1128/ AAC.02130-16 (2017)

18. Xu, W. et al. Chemical Genetic Interaction Profiling Reveals Determinants of Intrinsic Antibiotic Resistance in Mycobacterium tuberculosis. Antimicrob Agents Chemother 61, https://doi.org/10.1128/AAC.01334-17 (2017).

19. DeJesus, M. A., Ambadipudi, C., Baker, R., Sassetti, C. \& Ioerger, T. R. TRANSIT-A Software Tool for Himar1 TnSeq Analysis. PLoS Comput. Biol. 11, e1004401, https://doi.org/10.1371/journal.pcbi.1004401 (2015).

20. Belisle, J. T. et al. Role of the major antigen of Mycobacterium tuberculosis in cell wall biogenesis. Science 276, 1420-1422, https:// doi.org/10.1126/science.276.5317.1420 (1997)

21. Wu, C. W. et al. A novel cell wall lipopeptide is important for biofilm formation and pathogenicity of Mycobacterium avium subspecies paratuberculosis. Microb. Pathog. 46, 222-230, https://doi.org/10.1016/j.micpath.2009.01.010 (2009).

22. Wang, Q. et al. CpsA, a LytR-CpsA-Psr Family Protein in Mycobacterium marinum, Is Required for Cell Wall Integrity and Virulence. Infect. Immun. 83, 2844-2854, https://doi.org/10.1128/IAI.03081-14 (2015).

23. Wright, C. C. et al. The Mycobacterium tuberculosis MmpL11 Cell Wall Lipid Transporter Is Important for Biofilm Formation, Intracellular Growth, and Nonreplicating Persistence. Infect Immun 85, https://doi.org/10.1128/IAI.00131-17 (2017).

24. Namugenyi, S. B., Aagesen, A. M., Elliott, S. R. \& Tischler, A. D. Mycobacterium tuberculosis PhoY Proteins Promote Persister Formation by Mediating Pst/SenX3-RegX3 Phosphate Sensing. MBio 8, https://doi.org/10.1128/mBio.00494-17 (2017).

25. Khan, M. Z. et al. Protein kinase G confers survival advantage to Mycobacterium tuberculosis during latency-like conditions. J. Biol. Chem. 292, 16093-16108, https://doi.org/10.1074/jbc.M117.797563 (2017).

26. Paroha, R., Chourasia, R., Mondal, R. \& Chaurasiya, S. K. PknG supports mycobacterial adaptation in acidic environment. Mol. Cell Biochem. 443, 69-80, https://doi.org/10.1007/s11010-017-3211-x (2018).

27. Heller, J. L. E., Kamalampeta, R. \& Wieden, H. J. Taking a Step Back from Back-Translocation: an Integrative View of LepA/EF4's Cellular Function. Mol Cell Biol 37, https://doi.org/10.1128/MCB.00653-16 (2017).

28. Evans, R. N., Blaha, G., Bailey, S. \& Steitz, T. A. The structure of LepA, the ribosomal back translocase. Proc. Natl Acad. Sci. USA 105, 4673-4678, https://doi.org/10.1073/pnas.0801308105 (2008).

29. Qin, Y. et al. The highly conserved LepA is a ribosomal elongation factor that back-translocates the ribosome. Cell 127, 721-733, https://doi.org/10.1016/j.cell.2006.09.037 (2006).

30. Skye R. S. et al A conserved translation factor is required for optimal synthesis of a membrane protein family in mycobacteria Preprint at bioRxiv, https://doi.org/10.1101/872341 (2019).

31. Hicks, N. D., Carey, A. F., Yang, J., Zhao, Y. \& Fortune, S. M. Bacterial Genome-Wide Association Identifies Novel Factors That Contribute to Ethionamide and Prothionamide Susceptibility in Mycobacterium tuberculosis. MBio 10, https://doi.org/10.1128/ mBio.00616-19 (2019).

32. Hicks, N. D. et al. Clinically prevalent mutations in Mycobacterium tuberculosis alter propionate metabolism and mediate multidrug tolerance. Nat. Microbiol. 3, 1032-1042, https://doi.org/10.1038/s41564-018-0218-3 (2018).

33. Huff, J., Czyz, A., Landick, R. \& Niederweis, M. Taking phage integration to the next level as a genetic tool for mycobacteria. Gene 468, 8-19, https://doi.org/10.1016/j.gene.2010.07.012 (2010).

34. Baranowski, C. et al. Maturing Mycobacterium smegmatis peptidoglycan requires non-canonical crosslinks to maintain shape. Elife 7, https://doi.org/10.7554/eLife.37516 (2018).

\section{Acknowledgements}

B.J. and J.H.Z. conceived the study. B.W.W., J.H.Z. and B.J. designed research. B.W.W. and J.H.Z. performed research. B.W.W., J.H.Z. and B.J. analyzed the data. B.W.W. and B.J. wrote the manuscript with input from J.H.Z. This work was in part funded by grants from the Bill \& Melinda Gates Foundation (OPP1109789) and the National Natural Science Foundation of China (31570129) and start-up funds from Tsinghua University School of Medicine to B.J. B.J. is a Wellcome Trust Investigator $(207487 / \mathrm{B} / 17 / \mathrm{Z})$. The funders had no role in study design, data collection and interpretation, or the decision to submit the work for publication.

\section{Author contributions}

B.J. and J.H.Z. conceived the study. B.W.W., J.H.Z. and B.J. designed research. B.W.W. and J.H.Z. performed research. B.W.W., J.H.Z. and B.J. analyzed the data. B.W.W. and B.J. wrote the manuscript with input from J.H.Z. 


\section{Competing interests}

The authors declare no competing interests.

\section{Additional information}

Supplementary information is available for this paper at https://doi.org/10.1038/s41598-020-65308-2.

Correspondence and requests for materials should be addressed to B.J.

Reprints and permissions information is available at www.nature.com/reprints.

Publisher's note Springer Nature remains neutral with regard to jurisdictional claims in published maps and institutional affiliations.

(c) (1) Open Access This article is licensed under a Creative Commons Attribution 4.0 International License, which permits use, sharing, adaptation, distribution and reproduction in any medium or format, as long as you give appropriate credit to the original author(s) and the source, provide a link to the Creative Commons license, and indicate if changes were made. The images or other third party material in this article are included in the article's Creative Commons license, unless indicated otherwise in a credit line to the material. If material is not included in the article's Creative Commons license and your intended use is not permitted by statutory regulation or exceeds the permitted use, you will need to obtain permission directly from the copyright holder. To view a copy of this license, visit http://creativecommons.org/licenses/by/4.0/.

(C) The Author(s) 2020 